\section{Transrectal or transvaginal ultrasound- guided biopsy for pelvic masses: external validation and usefulness in oncologic patients}

\author{
So Yeon Won ${ }^{1}$, Hyun-Soo Kim², Sung Yoon Park ${ }^{1}$ \\ Departments of ${ }^{1}$ Radiology and ${ }^{2}$ Pathology, Yonsei University College of Medicine, Seoul, \\ Korea
}

Purpose: The purpose of this study was to externally validate the diagnostic usefulness of transrectal ultrasound (TRUS) or transvaginal ultrasound (TVUS)-guided biopsy for pelvic masses, and to analyze the diagnostic performance of these methods in oncologic patients.

Methods: A consecutive series of 30 patients who underwent TRUS- or TVUS-guided biopsy for pelvic masses was included. Tissue samples were obtained using an 18-gauge core biopsy needle under local anesthesia for lesions detected on computed tomography or magnetic resonance imaging. We analyzed the rates of diagnostic biopsies upon pathologic examination and biopsyrelated major complications requiring treatment. In diagnostic biopsy cases, the performance was also investigated for all patients and patients with underlying malignancy.

Results: The diagnostic biopsy rate was $93.3 \%$ (28 of 30 ) for all patients and 95.0\% (19 of 20) for oncologic patients. No patients had major complications. In diagnostic biopsies, the sensitivity, specificity, positive and negative predictive value, and accuracy for identifying malignancy were $100 \%$ ( 17 of 17 ), $72.7 \%$ (8 of 11 ), $85.0 \%$ (17 of 20 ), $100 \%$ ( 8 of 8 ), and $89.3 \%$ ( 25 of 28 ) for all patients and $100 \%$ (14 of 14 ), $60.0 \%$ (3 of 5), $87.5 \%$ (14 of 16), $100 \%$ (3 of 3), and $89.5 \%$ (17 of 19) for oncologic patients, respectively.

Conclusion: This study externally validated the feasibility and safety of TRUS- or TVUS-guided biopsy. In addition, these techniques appear to enable accurate pathologic diagnoses of pelvic masses in oncologic patients to be made safely and relatively noninvasively.

Keywords: Transrectal; Transvaginal; Ultrasound; Biopsy; Pelvis

\section{Introduction}

Percutaneous biopsy of intrapelvic lesions is difficult because various organs (e.g., small and large bowels, bladder and ureters, and uterus and ovaries) or large vessels usually surround the target lesion. Accordingly, risks of major organ injury or bleeding are associated with percutaneous biopsy. Therefore, for intrapelvic lesions, positron emission tomography/computed tomography (PET/CT) may be useful as the next step of computed tomography (CT) or magnetic resonance imaging (MRI) $[1,2]$.
ULTRA

SONO

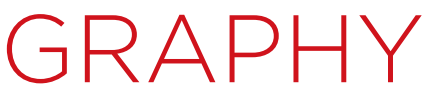

ORIGINAL ARTICLE

https://doi.org/10.14366/usg. 18028 pISSN: 2288-5919 - eISSN: 2288-5943 Ultrasonography 2019;38:149-155

Received: May 21, 2018

Revised: July 5, 2018

Accepted: July 23, 2018

Correspondence to:

Sung Yoon Park, MD, Department of Radiology, Yonsei University College of Medicine, 50 Yonsei-ro, Seodaemungu, Seoul 03722, Korea

Tel. +82-2-2228-7400

Fax. +82-2-393-3035

E-mail: sungyoon.park@samsung.com

This is an Open Access article distributed under the terms of the Creative Commons Attribution NonCommercial License (http://creativecommons.org/ licenses/by-nc/4.0/) which permits unrestricted noncommercial use, distribution, and reproduction in any medium, provided the original work is properly cited.

Copyright $\odot 2019$ Korean Society of Ultrasound in Medicine (KSUM)

How to cite this article:

Won SY, Kim HS, Park SY. Transrectal or transvaginal ultrasound-guided biopsy for pelvic masses: external validation and usefulness in oncologic patients. Ultrasonography. 2019 Apr;38(2):149-155. 
However, these imaging modalities are expensive and sometimes inaccurate, because some benign conditions mimic malignancy [3]. This may lead to the need for surgical confirmation as part of the planned management of a case when percutaneous biopsy is unavailable in practice.

Previous studies have suggested that transrectal ultrasound (TRUS) or transvaginal ultrasound (TVUS)-guided biopsy is useful and safe for sampling intrapelvic lesions [4-9]. According to the literature, the rate of diagnostic biopsies (i.e., those that enable a histologic diagnosis) is roughly $85 \%-95 \%$. The proximity between the TRUS or TVUS probe and deeply located intrapelvic lesions improves the sonic window and shortens the biopsy distance, which may result in a good diagnostic yield of those approaches $[4,10,11]$. However, the data showing the usefulness of TRUS- and TVUS-guided biopsy are still insufficient, and it is also unclear whether TRUS- or TVUS-guided biopsy is useful in oncologic patients.

Thus, we externally validated the diagnostic usefulness of TRUSor TVUS-guided biopsy for pelvic masses when performed by an experienced radiologist. We then also analyzed the diagnostic performance of these biopsy techniques in patients with an underlying malignancy.

\section{Materials and Methods}

\section{Patients}

This retrospective study was performed in accordance with the Declaration of Helsinki and approved by our Institutional Review Board. The requirement for informed consent was waved. We retrospectively reviewed a consecutive series of 30 patients (median age, 60.5 years) who underwent TRUS-guided $(n=19)$ or TVUSguided ( $n=11)$ biopsy for a pelvic mass between May 2014 and December 2017 at a single institution. All the patients had prebiopsy CT or MRI to demonstrate the target lesion for biopsy. The mean interval between the prebiopsy CT/MRI and biopsy was 8.5 days (range, 1 to 61 days).

Of the 30 patients, $20(66.7 \%)$ had an underlying malignancy (i.e., oncologic patients), belonging to the following categories: gynecologic cancer (e.g., uterine, cervical, endometrial, or vaginal cancers) $(n=7)$, colorectal cancer $(n=5)$, prostate cancer $(n=5)$, bladder cancer $(n=1)$, melanoma $(n=1)$, and nasopharyngeal cancer $(n=1)$. In this study, oncologic patients who underwent biopsy were defined as those who had a histologically proven primary cancer and underwent biopsy for a recurrent or metastatic lesion.

\section{TRUS- or TVUS-Guided Biopsy}

An experienced radiologist (S.Y.P.; 6 years' experience with TRUSand TVUS-guided biopsy) performed the biopsy. Local anesthesia with $10 \mathrm{~mL}$ of $1 \%$ lidocaine via a 21-gauge $15-\mathrm{cm}$ Chiba needle (Cook Medical, Bloomington, IN, USA) was administered to the rectal or vaginal mucosa. The biopsy was performed using an 18-gauge 20-cm biopsy gun (ACECUT, TSK Laboratory, Tochigi, Japan) under US guidance (Aixplorer US system, SuperSonic Imagine, Aix-en-Provence, France). The left lateral decubitus position was used for the TRUS approach and the lithotomy position was applied for the TVUS approach. Prophylactic antibiotics (e.g., second or third-generation cephalosporin, or fluoroquinolone) were administered intravenously before biopsy and orally after biopsy for 7 days because the biopsy routes, which involved the rectal or vaginal canal, were not completely aseptic. In addition, a prebiopsy enema with glycerin solution was conducted 1-2 hours before TRUS-guided biopsy. All patients were discharged 4-6 hours after the biopsy to monitor immediate complications. No patients were hospitalized for the biopsy.

\section{Radiologic, Pathologic, and Clinical Data Analyses}

All CT, MRI, and ultrasonography (US) images were archived using a picture archiving and communication system (PathSpeed, GE Healthcare, Milwaukee, WI, USA) for the analysis. Two radiologists (S.Y.P. and S.Y.W.) evaluated the pelvic lesions in consensus with prebiopsy CT or MRI as follows: (1) lesion size, (2) lesion nature (e.g., solid or cystic), and (3) lesion location (e.g., perirectal space, uterus or ovary, vaginal vault, pelvic sidewall, or bladder). A lesion was considered to have a cystic nature when its non-enhancing cystic or necrotic portions comprised over half of the lesion. On the basis of US images and reports, the biopsy core number and biopsy distance were assessed. The biopsy distance was defined by the mean length of the biopsy needle seen on US images.

The final pathologic report of the biopsy specimens from an experienced pathologist (H.S.K.; 4 years' experience) was our reference standard to determine whether the biopsy result was diagnostic or non-diagnostic, and whether the lesion was malignant or benign. We compared the radiologic impressions obtained through CT or MRI and the findings of the prebiopsy US examination with the pathologic result of the biopsy specimen.

Major complications were investigated based on the patients' medical records. Major complications were defined as those with a Clavien-Dindo classification of 2 or greater (i.e., complications requiring pharmacologic, interventional, or surgical treatment) [12].

\section{Statistical Analysis}

We calculated the rate of diagnostic biopsies. For patients with diagnostic results, the sensitivity, specificity, positive predictive value (PPV), negative predictive value (NPV), and accuracy of TRUS- or TVUS-guided biopsy in diagnosing the malignancy were assessed. 
These measures of diagnostic performance were also analyzed in patients with an underlying malignancy.

\section{Results}

\section{Radiologic and Biopsy-Related Characteristics}

For the 30 pelvic lesions in 30 patients, the median lesion size was $4.5 \mathrm{~cm}$ (range, 0.9 to $13.0 \mathrm{~cm}$ ). The lesions had a solid nature in 25 patients and a cystic nature in 5 . The most common location of the biopsied lesions was the perirectal space $(n=10)$, followed by gynecologic organs (Table 1). Of the 30 patients, $20(66.7 \%)$ had an underlying malignancy. The median number of biopsy cores was 4 (range, 2 to 7). The median biopsy distance was $2.4 \mathrm{~cm}$ (range, 1.4 to $5.6 \mathrm{~cm})$

\section{Biopsy Performance and Major Complications}

The overall diagnostic rate of TRUS- or TVUS-guided biopsy was $93.3 \%$ (28 of 30). The diagnostic biopsies revealed 17 malignant and 11 benign lesions. The biopsy-proven malignant lesions consisted of recurrent gynecologic cancer $(n=4)$ (Fig. 1), recurrent prostate cancer $(n=4)$, recurrent colorectal cancer $(n=3)$ (Fig.

Table 1. Characteristics of the patients included in the study

\begin{tabular}{|c|c|}
\hline Parameter & Value \\
\hline \multicolumn{2}{|l|}{ Patients characteristics } \\
\hline Age (yr) & $60.5(23-89)$ \\
\hline Sex (male: female) & $12: 28$ \\
\hline Proportion of oncologic patients, n (\%) & $20(66.7)$ \\
\hline \multicolumn{2}{|l|}{ Radiologic characteristics } \\
\hline Prebiopsy imaging, CT: MRI & $14: 16$ \\
\hline Lesion size $(\mathrm{cm})$ & $4.5(0.9-13.0)$ \\
\hline Lesion nature (solid:cystic) & $25: 5$ \\
\hline \multicolumn{2}{|l|}{ Lesion location } \\
\hline Perirectal space & 10 \\
\hline Uterus or ovary & 7 \\
\hline Vaginal vault & 5 \\
\hline Pelvic sidewall & 5 \\
\hline Bladder & 3 \\
\hline \multicolumn{2}{|l|}{ Biopsy characteristics } \\
\hline Interval between imaging and biopsy (day) & $8.5(1-61)$ \\
\hline Approach type (TRUS:TVUS) & 19:11 \\
\hline Biopsy core number & $4.0(2-7)$ \\
\hline Biopsy distance $(\mathrm{cm})$ & $2.4(1.4-5.6)$ \\
\hline
\end{tabular}

Data of age, lesion size, interval between imaging and biopsy, biopsy core number, and biopsy distance are median (range).

$\mathrm{CT}$, computed tomography; MRI, magnetic resonance imaging; TRUS, transrectal ultrasound; TVUS, transvaginal ultrasound.
Table 2. Performance of TRUS- and TVUS-guided biopsy in diagnosing malignancy

\begin{tabular}{lcrc}
\hline \multicolumn{1}{c}{ Parameter } & Overall $(\mathrm{n}=28)$ & TRUS $(\mathrm{n}=18)$ & \multicolumn{1}{c}{ TVUS $(\mathrm{n}=10)$} \\
\hline Sensitivity & $17 / 17(100)$ & $13 / 13(100)$ & $4 / 4(100)$ \\
Specificity & $8 / 11(72.7)$ & $5 / 5(100)$ & $3 / 6(50.0)$ \\
PPV & $17 / 20(85.0)$ & $13 / 13(100)$ & $4 / 7(57.1)$ \\
NPV & $8 / 8(100)$ & $5 / 5(100)$ & $3 / 3(100)$ \\
Accuracy & $25 / 28(89.3)$ & $18 / 18(100)$ & $7 / 10(70.0)$ \\
\hline
\end{tabular}

Values are presented as number (\%).

Of the 30 patients, two with non-diagnostic results were excluded from the analysis of diagnostic performance for malignancy because of the unclear information regarding malignancy or benignity.

TRUS, transrectal ultrasound; TVUS, transvaginal ultrasound; PPV, positive predictive value; NPV, negative predictive value.

2), lymphoma $(n=2)$, recurrent bladder cancer $(n=1)$, recurrent nasopharyngeal cancer $(n=1)$, recurrent melanoma $(n=1)$, and primary ovarian cancer before neoadjuvant chemotherapy $(n=1)$. The biopsy-proven benign lesions consisted of inflammation $(n=6)$, uterine leiomyoma $(n=2)$, ganglioneuroma $(n=1)$, lymphangioma $(n=1)$, and retroperitoneal fibrosis $(n=1)$.

For the diagnostic biopsies, the sensitivity, specificity, PPV, NPV, and accuracy of overall TRUS- or TVUS-guided biopsy for identifying malignancy were $100 \%$ (17 of 17), $72.7 \%$ (8 of 11 ), $85.0 \%$ (17 of 20 ), $100 \%$ ( 8 of 8 ), and $89.3 \%$ ( 25 of 28 ), respectively (Table 2). No patient had major complications requiring pharmacologic, interventional, or surgical treatment.

The diagnostic rates of TRUS and TVUS were $94.4 \%$ (17 of 18) and $90.0 \%$ (9 of 10), respectively. The sensitivity, specificity, PPV, NPV, and accuracy of TRUS-guided biopsy were all 100\% for diagnosing malignancy, while the corresponding values of TVUSguided biopsy were $100 \%$ (4 of 4), 50.0\% (3 of 6 ), $57.1 \%$ (4 of 7), $100 \%$ (3 of 3), and $70.0 \%$ (7 of 10$)$, respectively.

For the 20 oncologic patients, the overall diagnostic biopsy rate was $95.0 \%$ (19 of 20). For the diagnostic biopsies, the sensitivity, specificity, PPV, NPV, and accuracy were 100\% (14 of 14), $60.0 \%$ (3 of 5), $87.5 \%$ (14 of 16 ), 100\% (3 of 3), and $89.5 \%$ (17 of 19 ), respectively, for diagnosing malignancy.

\section{Non-diagnostic Cases}

In this study, two patients had non-diagnostic results. One patient (age, 86 years old; female) had a uterine mass, measuring 10.5 $\mathrm{cm}$ in size (Fig. 3). On contrast-enhanced CT, the lesion had large intratumoral cystic or necrotic portions without enhancement (i.e., it was cystic in nature). TVUS-guided biopsy with seven cores was performed under the impression of primary uterine sarcoma, and the pathologic result was non-diagnostic because 


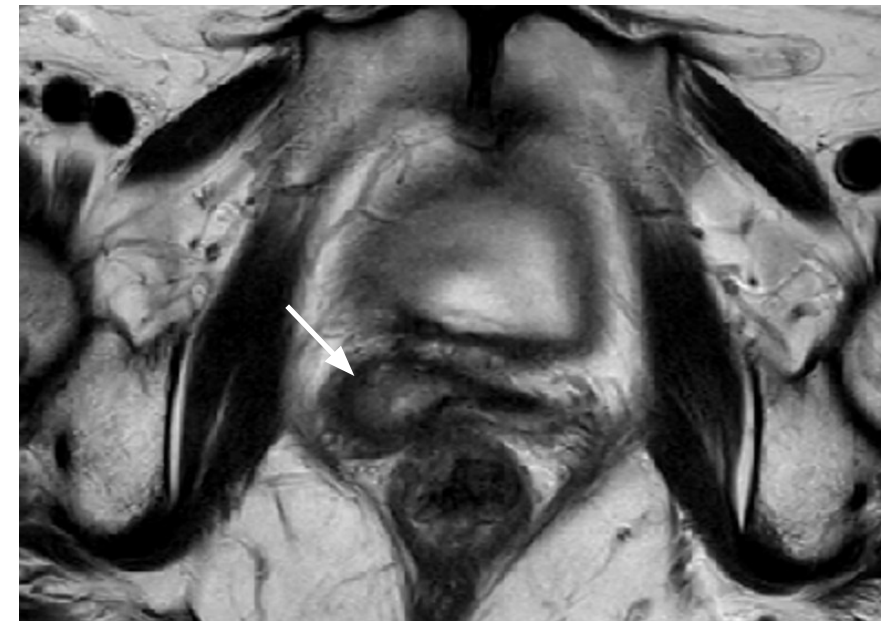

A

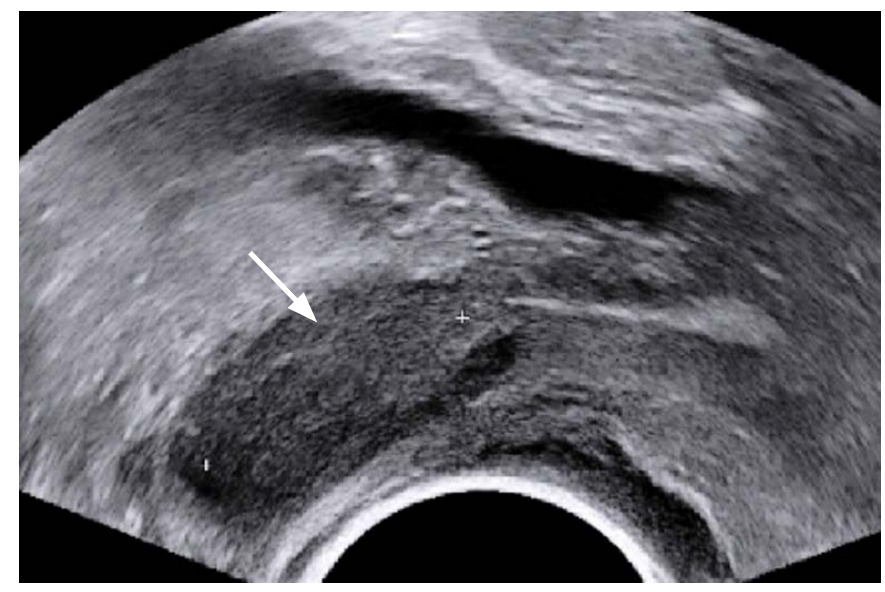

C



B

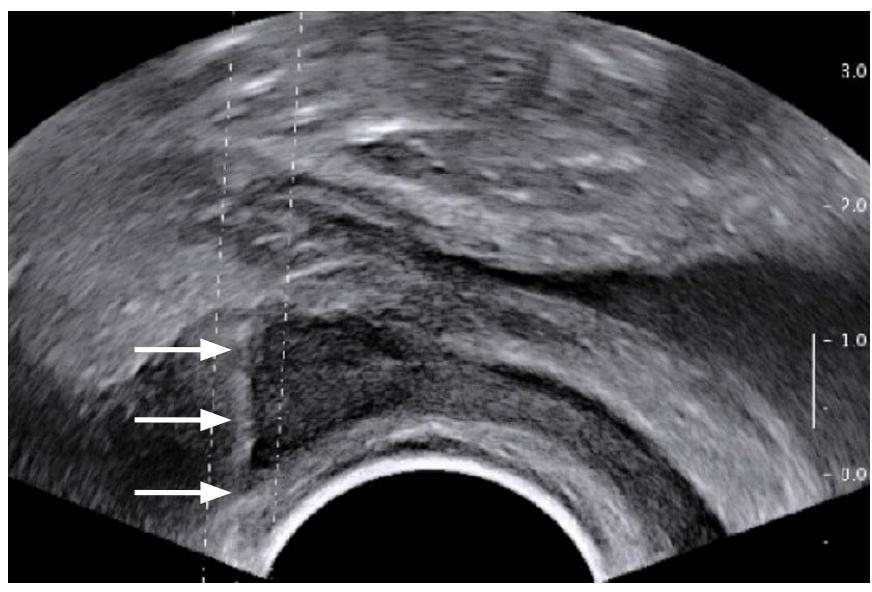

D

Fig. 1. A 58-year-old woman who had a history of surgery for endometrial cancer.

A, B. Axial T2-weighted (A) and fat saturation contrast-enhanced T1-weighted (B) images depict a solid mass, measuring $2.1 \mathrm{~cm}$, in the vaginal vault (arrows). C. Transverse transvaginal ultrasound (TVUS) image also demonstrates a hypoechoic mass at the corresponding site (arrow). D. TVUS-guided core biopsy (arrows) with 4 cores reveals recurrent cancer.

the biopsied specimens had only necrotic tissues surrounded by fibrous connective tissue without cancer cells. This patient was lost to follow-up after the biopsy. The other patient (age, 74 years old; male) had a focal solid mass, measuring $3.6 \mathrm{~cm}$, near the pelvic sidewall. TRUS-guided biopsy with 5 cores was performed under the impression of recurrent prostate cancer. The pathologic examination indicated that the biopsy was non-diagnostic because only nonspecific muscle was found. The lesion had increased in size on follow-up CT taken 1 year later and radiation therapy was performed on the basis of the radiologic findings.

\section{Discussion}

In this study, we analyzed the diagnostic performance and safety of
TRUS- or TVUS-guided biopsy for pelvic masses. The diagnostic rate (i.e., the proportion of biopsies that allowed a specific pathologic diagnosis) was greater than $90 \%$ for all patients and for oncologic patients, while there were no major complications requiring treatment. In addition, these biopsy techniques seemed to be highly sensitive for identifying malignancy, as shown by the sensitivity of $100 \%$ for all patients and for oncologic patients. Therefore, in oncologic patients, TRUS- or TVUS-guided biopsy may be very useful for safely and accurately identifying cases of pelvic metastasis or recurrence.

The sonic window is generally poor when the distance between the US probe and targeted region increases. In addition, acoustic shadowing from the bowels in the pelvic cavity often makes the target lesion invisible. Thus, visualizing deep pelvic masses using 


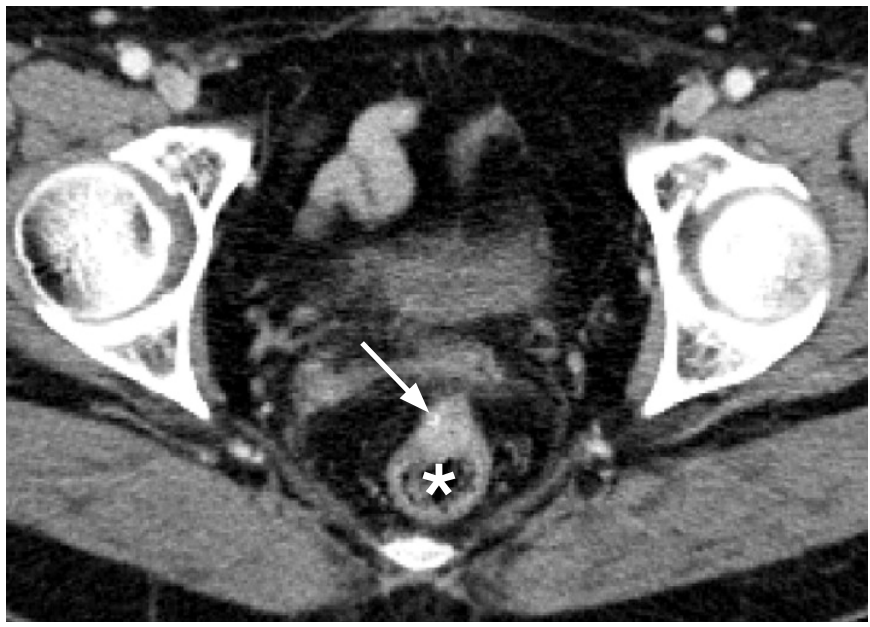

A

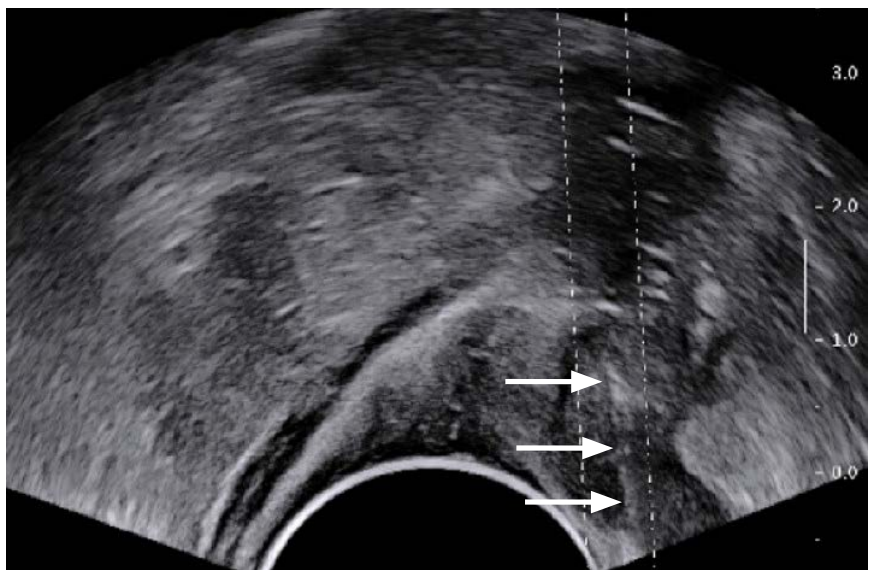

C

transabdominal US is more difficult than visualizing superficially located lesions [10]. However, the distance between deep pelvic masses and the TRUS or TVUS probe is short because the proximal vagina or rectum is usually located in the central portion of the pelvic cavity $[4,11]$. In this study, the median biopsy distance was $2.4 \mathrm{~cm}$, which may allow a good sonic window, subsequently resulting in accurate and safe tissue sampling, with the concomitant avoidance of adjacent organ injury.

In oncologic patients, the accurate diagnosis of recurrent disease is crucial before treatment planning. In daily practice, CT and MRI are important imaging modalities for initially detecting pelvic recurrence. However, the radiologic features of recurrent disease and postoperative benign lesions sometimes overlap [13]. In patients who have undergone hysterectomy for a gynecologic malignancy, differentiating between recurrent disease and primary vaginal cancer may also be difficult radiologically [14]. PET/CT, as a problem-solving tool, also has some limitations in lesion characterization, leading to false positive or negative results $[3,15,16]$, and it is expensive. For

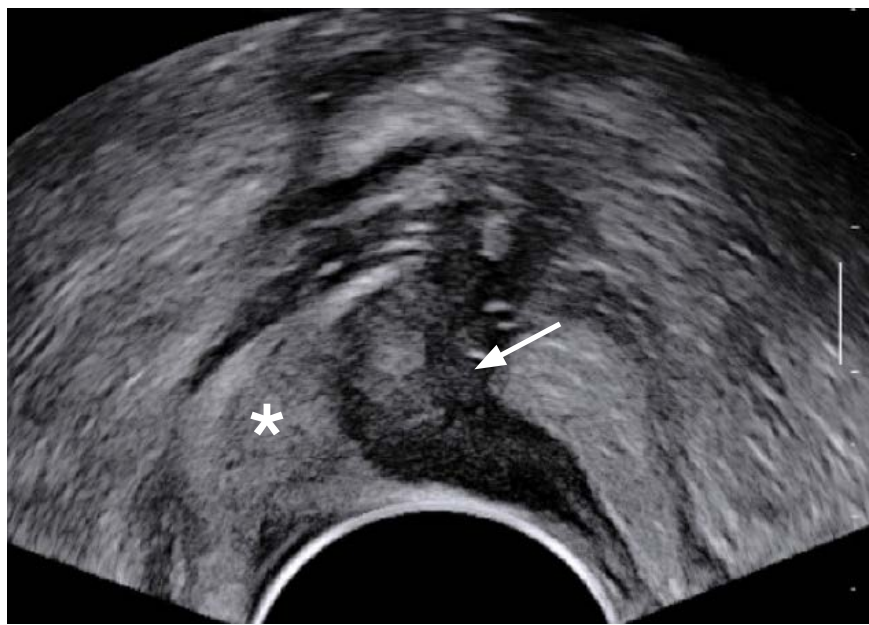

B

Fig. 2. A 63-year-old woman who had a history of surgery for sigmoid colon cancer.

A. Contrast-enhanced computed tomography image depicts a solid mass (arrow), measuring $1.8 \mathrm{~cm}$, abutting the anterior wall of the rectum (asterisk). B. Longitudinal transrectal ultrasound (TRUS) image also demonstrates a hypoechoic mass (arrow) at the anterior wall of the rectum (asterisk). C. TRUS-guided core biopsy (arrows) with three cores reveals recurrent cancer.

the patients with a radiologically indeterminate pelvic lesion, TRUSor TVUS-guided biopsy may be useful to plan treatment strategies.

Uncertainty remains regarding the necessity of prophylactic antibiotics. Theoretically, the transrectal and transvaginal approaches are not completely aseptic. For prostate biopsy, antibiotic prophylaxis is well-established when the transrectal approach is used [17]. However, whether antibiotic prophylaxis is necessary for TVUS-guided biopsy remains controversial $[4,9]$. Nevertheless, previous reports imply that transvaginal procedures may have a risk of infectious complications $[18,19]$. Endovaginal flora or other pathogens may be transmitted into the pelvic cavity by a contaminated TVUS probe or biopsy needle [20]. For this reason, antibiotic prophylaxis was applied in this study for both TRUS- and TVUS-guided biopsies. More studies are necessary to determine the optimal preparations for biopsy.

This study has limitations. First, a single experienced operator performed the biopsies. The diagnostic performance of TRUSand TVUS-guided biopsy should be tested in radiologists with 


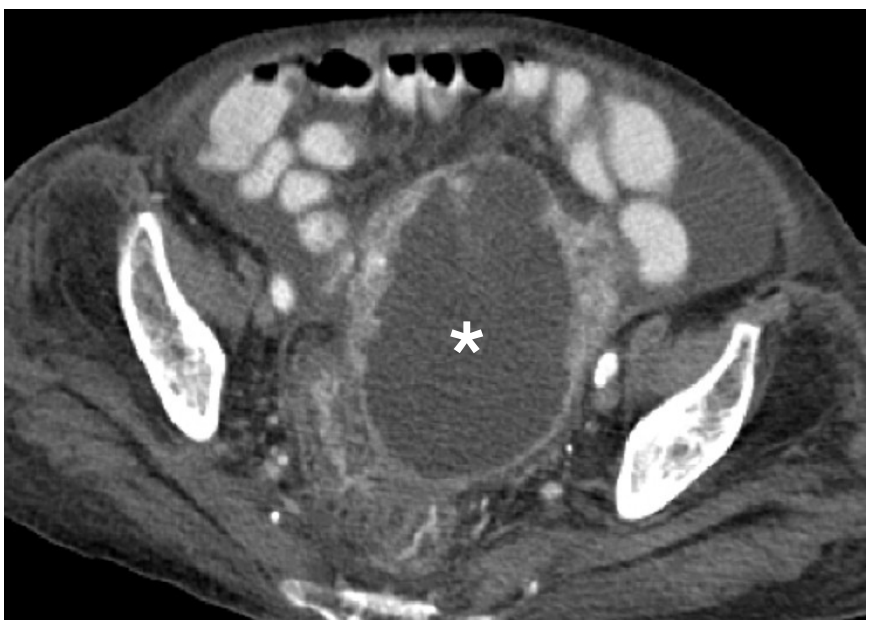

A

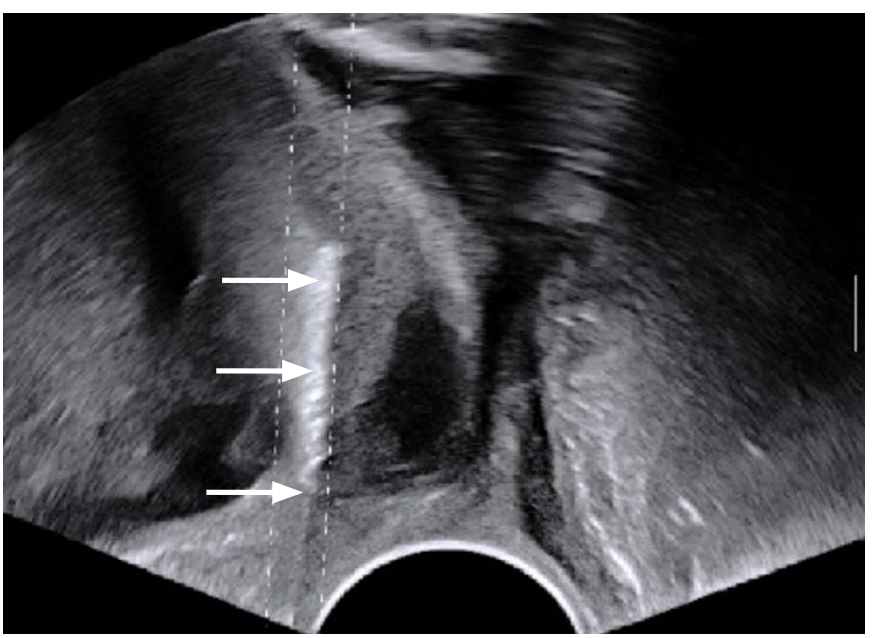

C

various levels of experience with biopsy, which may provide useful information with respect to the learning curve and suggest the optimal threshold of experience. Second, a comparison between diagnostic and non-diagnostic biopsies was not made due to the limited statistical power, as only two patients had non-diagnostic results. Third, there was a risk of sampling error in the biopsies, because core biopsies might sample only the regions of peritumoral inflammation. Thus, clinical and imaging follow-up is necessary to reduce the likelihood of underestimation when the biopsy results for a lesion with a high suspicion of malignancy on imaging are nondiagnostic or suggestive of benignity.

In conclusion, this study externally validated the feasibility and safety of TRUS- or TVUS-guided biopsy. In addition, these techniques appear to enable accurate pathologic diagnoses of pelvic masses in oncologic patients to be made safely and relatively noninvasively.

ORCID: Sung Yoon Park: https://orcid.org/0000-0003-0996-7595

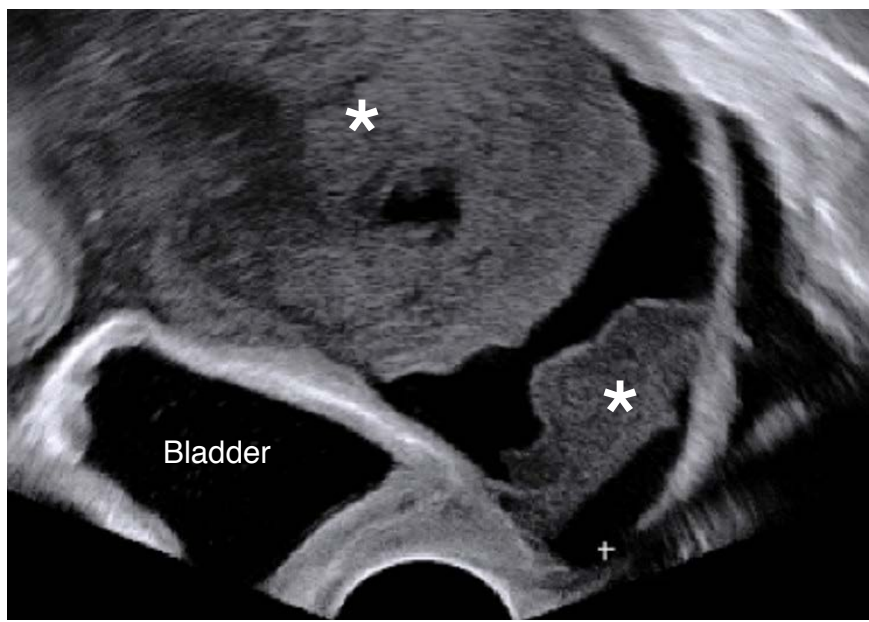

B

Fig. 3. An 86-year-old woman who had a uterine mass.

A. Contrast-enhanced computed tomography image depicts a cystic or necrotic mass (asterisk), measuring $10.5 \mathrm{~cm}$, in the pelvic cavity. B. Longitudinal transvaginal ultrasound (TVUS) image also demonstrated a mass (asterisks) at the corresponding site of the uterus. C. TVUS-guided core biopsy with seven cores was performed in the echogenic portions of the mass (arrows), and it was nondiagnostic pathologically.

\section{Conflict of Interest}

No potential conflict of interest relevant to this article was reported.

\section{References}

1. Even-Sapir E, Parag Y, Lerman H, Gutman M, Levine C, Rabau $M$, et al. Detection of recurrence in patients with rectal cancer: $\mathrm{PET} / \mathrm{CT}$ after abdominoperineal or anterior resection. Radiology 2004:232:815-822.

2. Yamamoto $Y$, Oguri $H$, Yamada R, Maeda N, Kohsaki S, Fukaya T. Preoperative evaluation of pelvic masses with combined $18 \mathrm{~F}$-fluorodeoxyglucose positron emission tomography and computed tomography. Int J Gynaecol Obstet 2008;102:124-127.

3. Subhas N, Patel PV, Pannu HK, Jacene HA, Fishman EK, Wahl RL. Imaging of pelvic malignancies with in-line FDG PET-CT: case examples and common pitfalls of FDG PET. Radiographics 2005;25:1031-1043. 
4. Park JJ, Kim CK, Park BK. Ultrasound-guided transvaginal core biopsy of pelvic masses: feasibility, safety, and short-term followup. AJR Am J Roentgenol 2016;206:877-882.

5. Lin $S Y$, Xiong YH, Yun $M$, Liu LZ, Zheng $W$, Lin $X$, et al. Transvaginal ultrasound-guided core needle biopsy of pelvic masses. J Ultrasound Med 2018;37:453-461.

6. Savader BL, Hamper UM, Sheth S, Ballard RL, Sanders RC. Pelvic masses: aspiration biopsy with transrectal US guidance. Radiology 1990;176:351-353.

7. Rinnab L, Kufer R, Hautmann RE, Gottfried HW. Use of transrectal ultrasound-guided biopsy in the diagnosis of pelvic malignancies. J Clin Ultrasound 2006;34:440-445.

8. Dadayal G, Weston M, Young A, Graham JL, Mehta K, Wilkinson $\mathrm{N}$, et al. Transvaginal ultrasound (TVUS)-guided biopsy is safe and effective in diagnosing peritoneal carcinomatosis and recurrent pelvic malignancy. Clin Radiol 2016;71:1184-1192.

9. Plett SK, Poder L, Brooks RA, Morgan TA. Transvaginal ultrasoundguided biopsy of deep pelvic masses: how we do it. J Ultrasound Med 2016;35:1113-1122.

10. Park BK. Ultrasound-guided genitourinary interventions: principles and techniques. Ultrasonography 2017;36:336-348.

11. O'Neill MJ, Rafferty EA, Lee SI, Arellano RS, Gervais DA, Hahn PF, et al. Transvaginal interventional procedures: aspiration, biopsy, and catheter drainage. Radiographics 2001;21:657-672.

12. Dindo D, Demartines N, Clavien PA. Classification of surgical complications: a new proposal with evaluation in a cohort of 6336 patients and results of a survey. Ann Surg 2004;240:205-213.

13. Vargas HA, Wassberg $C$, Akin 0 , Hricak H. MR imaging of treated prostate cancer. Radiology 2012;262:26-42.

14. Parikh JH, Barton DP, Ind TE, Sohaib SA. MR imaging features of vaginal malignancies. Radiographics 2008;28:49-63.

15. Long NM, Smith CS. Causes and imaging features of false positives and false negatives on F-PET/CT in oncologic imaging. Insights Imaging 2011;2:679-698.

16. Blake MA, Singh A, Setty BN, Slattery J, Kalra M, Maher MM, et al. Pearls and pitfalls in interpretation of abdominal and pelvic PET-CT. Radiographics 2006;26:1335-1353.

17. Mottet N, Bellmunt J, Bolla M, Briers E, Cumberbatch MG, De Santis $\mathrm{M}$, et al. EAU-ESTRO-SIOG guidelines on prostate cancer. Part 1: screening, diagnosis, and local treatment with curative intent. Eur Urol 2017;71:618-629.

18. Curtis P, Amso N, Keith E, Bernard A, Shaw RW. Evaluation of the risk of pelvic infection following transvaginal oocyte recovery. Hum Reprod 1991;6:1294-1297.

19. Mikamo H, Kawazoe K, Sato Y, Itoh M, Tamaya T. Ovarian abscess caused by Peptostreptococcus magnus following transvaginal ultrasound-guided aspiration of ovarian endometrioma and fixation with pure ethanol. Infect Dis Obstet Gynecol 1998;6:66-68.

20. M'Zali F, Bounizra C, Leroy S, Mekki Y, Quentin-Noury C, Kann M. Persistence of microbial contamination on transvaginal ultrasound probes despite low-level disinfection procedure. PLoS One 2014;9:e93368. 